\title{
Psychosocial interventions for reducing depression among the elderly in low and middle income countries: A Systematic Review Protocol
}

Jordan Mutambi Amanyire

Mbarara University of Science and Technology

Irene Aheisibwe

Bishop Stuart University

Godfrey Zari Rukundo ( $\nabla$ grukundo@must.ac.ug )

Mbarara University of Science and Technology https://orcid.org/0000-0002-3739-1426

\section{Protocol}

Keywords: Depression, elderly, psychosocial interventions

Posted Date: February 12th, 2021

DOI: https://doi.org/10.21203/rs.3.rs-74810/v2

License: (c) (i) This work is licensed under a Creative Commons Attribution 4.0 International License.

Read Full License 


\section{Abstract}

Background: According to the World Health Organization, depression is expected to be the largest contributor to the global disease burden by 2030. Depression is the most frequent cause of emotional distress and reduced quality of life among older people affecting over $12 \%$ of the individuals aged 65 or older, . Psychosocial interventions have been proven to be effective in the management of depression. Most of the available evidence is from high income settings, with paucity of information in low and middle income countries which carry the biggest burden of depression and other health challenges. In this systematic review, we will document evidence on psychosocial interventions that have been effective in treatment of depression among elderly people in low and middle income countries.

Methods: The review will be conducted and reported in accordance to the preferred reporting items for systematic reviews and meta-analysis (PRISMA) statement. We will include journal articles that have documented the psychosocial interventions in the primary prevention of depressive symptoms in elderly people aged 60 or older. We will also include accessible grey literature about the topic. We will include articles that have documented the psychosocial interventions to address depression in elderly patients in low and middle income countries. We will search different search engines and data bases including PubMed, EMBASE, Psych-INFO, Cochrane Library. We will use a meta-analysis, should we find that there is no heterogeneity between included studies.

Discussion: This protocol describes a planned systematic review of observational studies reporting psychosocial interventions in the management of depressive symptoms in elderly people aged 60 or older. We anticipate that once this review is complete and published, our findings will be of interest to the elderly with depressive symptoms, their families and caregivers, students, and other healthcare professionals, scientists and policy makers.

Systematic review registration: This protocol will not be registered with PROSPERO International prospective register of systematic reviews since the system is no longer accepting new protocols.

\section{Background}

According to the World Health Organization [2017], depression is expected to be the largest contributor to the global disease burden by 2030 (1). Among the mental disorders that affect older people, depression is the most frequent cause of emotional distress and reduced quality of life and is recognized (2). Among individuals aged 65 or older, over $12 \%$ are affected by depressive syndromes (3-6). The prevalence of depressive disorders is substantially higher among specific populations of older persons, ranging from 5$10 \%$ in medical outpatients to $14-42 \%$ in residents of long-term care facilities $(7,8)$.

Given the increasing population of the elderly, depression in older adults may increase as well, and as such, effective interventions are needed to prevent depression and also manage depressive symptoms. Psychosocial interventions have been proven to be effective in the management of depression in different age groups $(9,10)$. Such interventions emphasize psychological or social rather than biological 
factors. Most of the available evidence is from high income settings, with paucity of information in low and middle income countries which carry the biggest burden of depression and other health challenges. There is need to document evidence on psychosocial interventions among the elderly in low and middle income countries.

\section{Objective}

The objective of this systematic review is to document evidence on psychosocial interventions that have been effective in treatment of depression among elderly (60 years and older) people in low and middle income countries.

\section{Methods}

The review will be conducted and reported in accordance to the preferred reporting items for systematic reviews and meta-analysis (PRISMA) statement $(11,12)$. This systematic review protocol title will be registered with the International Prospective Register of Systematic Reviews (PROSPERO).

\section{Eligibility criteria}

We will include articles that have documented the psychosocial interventions to address depression in elderly patients in low and middle income countries.

Psychosocial interventions are defined as any interventions that emphasize psychological or social factors rather than biological factors (13). This definition allows for the inclusion of psychological therapies and health education as well as interventions with a focus on social aspects, such as social support and networking. Interventions with a physiological component in addition to a psychosocial component (e.g., exercise groups for older people) will also be considered.

The psychosocial interventions could appear in any format, for example, in groups or individually, as long as they are described in the study and would allow for replication. Interventions with organization of care as the main focus will not be considered for this review. All trials of secondary and tertiary preventive interventions, relapse prevention, and pharmacological interventions will be excluded.

The review will include articles from studies done following cross-sectional, longitudinal, cohort, case control and randomized control study designs. Sub-group analysis will be performed on the included studies to cater for the variations in the study designs.

Unpublished but accessible studies will be included in the review. Studies on non-suicidal deliberate selfharm will be excluded. Articles with only abstracts available (with no full articles) will be excluded as they may be difficult to compare with full articles.

The selection criteria of the articles for inclusion will be strictly applied and no double reporting of the same outcome will be included in the review. We will report the study as a single one, but in the event that 
there could be separate outcomes, we will report them as such. Each publication will have the name of the author, year, title and publisher.

The trials to be considered for this review will have depressive symptoms or depression as a measured outcome. Furthermore, to be eligible, trials will have to encompass a control condition: either care as usual, waiting list, or no intervention.

\section{Study design}

We will conduct a systematic review of observational studies (cross-sectional, cohort, longitudinal and case control studies) that documented psychosocial interventions to address depression in elderly patients in low and middle income countries. We will also describe the most common psychosocial interventions for depression, identify factors that promote psychosocial interventions for depression and assess the effectiveness of psychosocial interventions for depression among elderly patients.

\section{Setting}

The systematic review will only include studies conducted in low and middle income countries all over the World.

\section{Participants}

The participants in this systematic review will be elderly persons aged 50 years and above.

\section{Comparisons}

The comparison groups will be elderly provided with psychosocial interventions and those without (biological care).

\section{Outcomes}

The primary outcome of the review will be the occurrence of depression and depressive symptoms, as measured by Depression Rating Scales. Secondary outcomes will be functional level and quality of life. The outcome measures will be recorded immediately after the intervention and at end of follow-up.

\section{Search methods}

The search strategy will be carried out by the research team using the following electronic databases and search engines, from inception, using the same search strategy (Appendix A) with alterations as appropriate for each database: the Cochrane Library, PsychINFO, PubMed, EMBASE, Africa wideinformation and global health. We will hand search the references of the included studies. We will use a combination of mesh terms, text words and combine them with appropriate Boolen operators in order to identify as many studies as possible. Grey literature will also be included. We will identify the relevant grey literature using web searching, web-based catalogues as well as using bibliographic databases. 


\section{Data extraction}

All publications retrieved from the databases will be screened for inclusion by the primary reviewer and an independent researcher separately. Available data will be extracted and coded independently by the primary reviewer and a second data extractor.

The methodological quality of the included intervention studies will be assessed and rated according to the Cochrane Collaboration Handbook (14). Quality of the study will be assessed through risk of bias assessment tool which will be done using a checklist developed from the strobe statement [16].

The data collected will also include the authors' names, the title of the article and the year the study was conducted. This data will be extracted by two content experts on the research team. For articles with missing information, the primary authors will be contacted by the principle investigator to provide the missing or additional data. For any discrepancies, the principle investigator will make the final decision. Data extraction will be done in two stages by four people of different specialties that include a Psychiatrist, Psychologist Statistician and an expert in reviewing systematic reviews. We will then download the full text articles for further screening by independent reviewers who will be identified. In the event that there is disagreement about which study to include or exclude, the PI will be the arbitrator.

\section{Quality assessment}

All publications retrieved from the databases will be screened for inclusion by the primary reviewer. Available data will be extracted and coded independently by the primary reviewer and the systematic review expert.

The methodological quality of the included intervention studies will be assessed and rated according to the Cochrane Collaboration Handbook (14). Specifically, the Cochrane risk of bias (RoB) tool will be used to assess the quality of research papers. The studies will be rated by taking five individual domains into consideration (sequence generation; allocation concealment; personnel, and outcome assessors; incomplete outcome data; selective outcome reporting; and other sources of bias) and giving them a quality rating of "low risk of bias," "unclear," or "high risk of bias." We will use GRADE criteria to assess the quality of evidence. Specifically, we will consider risk of bias, imprecision, indirectness, inconsistency and possible publication bias.

\section{Outcomes Measured}

The primary outcome of the review will be the occurrence of depression and depressive symptoms, as measured by depression rating scales (such as the Geriatric Depression Scale). Secondary outcomes will be functional level and quality of life. The outcome measures will be recorded either immediately after the intervention or at end of follow-up. 


\section{Data analysis}

Data will be entered into Review Manager 5.0 software by the principal reviewer and systematic review expert separately. For binary efficacy outcomes (e.g., depression), the Mantel-Haenszel random effects model for calculating odds ratio (OR) will be applied. Where intention-to treat (ITT) data will not be available, end-point continuous data for trial completers will be used. For continuously distributed outcomes, the Weighted Mean Difference (WMD) or Standardized Mean Difference (SMD) will be calculated as appropriate using a random effects model. SMD will be calculated when outcomes will be measured using different scoring systems.

If measures of variance of outcomes are found through publications, through calculations, or by contacting the authors, the outcome will be excluded from the meta-analysis. Substantially, skewed data (where the standard deviation is more than twice the mean value) will not be entered in the meta-analysis. The impact of statistical heterogeneity on the meta-analysis will be assessed by quantifying inconsistency among the studies with the 12 Index test [15]. Sensitivity analyses will be conducted by looking at randomized trials only and at studies with low risk of bias only (i.e., at least two out of five domains will be rated as low risk of bias for the study).

\section{Abbreviations}

ITT Intention to treat

PI Principal Investigator

PRISMA Preferred reporting items for systematic reviews and meta-analysis

\section{Declarations}

\section{Ethics approval and consent to participate}

No ethical approval will be required for the performance of this systematic review

\section{Consent for publication}

No need for consent for publication. No raw data will be used. We will use already published data.

\section{Availability of data and material}

Not applicable

\section{Competing interests}

The authors declare that they have no competing interests. 


\section{Funding}

This research received no specific grant from any funding agency in the public, commercial or non-forprofit sectors.

\section{Author contributions}

All authors have significantly contributed to the writing of this protocol.

\section{References}

1. WHO. Depression and Other Common Mental Disorders Global Health Estimates. Geneva, Switzerland.: World Health Organisation; 2017.

2. Blazer DG. Depression in late life: review and commentary. J Gerontol A Biol Sci Med Sci. 2003;58(3):249-65.

3. Luijendijk HJ, van den Berg JF, Dekker MJ, van Tuijl HR, Otte W, Smit F, et al. Incidence and recurrence of late-life depression. Arch Gen Psychiatry. 2008;65(12):1394-401.

4. WHO. Global burden of disease: 2004 update. Geneva, Switzerland: World Health Organization; 2008.

5. Copeland JR, Beekman AT, Dewey ME, Hooijer C, Jordan A, Lawlor BA, et al. Depression in Europe. Geographical distribution among older people. Br J Psychiatry. 1999;174:312-21.

6. Forsman AK, Schierenbeck I, Wahlbeck K. Psychosocial interventions for the prevention of depression in older adults: systematic review and meta-analysis. J Aging Health. 2011;23(3):387416.

7. Fiske A, Wetherell JL, Gatz M. Depression in older adults. Annu Rev Clin Psychol. 2009;5:363-89.

8. Hybels CF, Pieper CF, Blazer DG, Steffens DC. The course of depressive symptoms in older adults with comorbid major depression and dysthymia. Am J Geriatr Psychiatry. 2008;16(4):300-9.

9. Priebe S, Fung C, Sajun SZ, Alinaitwe R, Giacco D, Gomez-Restrepo C, et al. Resource-oriented interventions for patients with severe mental illnesses in low- and middle-income countries: trials in Bosnia-Herzegovina, Colombia and Uganda. BMC Psychiatry. 2019;19(1):181.

10. Reynolds CF, 3rd, Cuijpers P, Patel V, Cohen A, Dias A, Chowdhary N, et al. Early intervention to reduce the global health and economic burden of major depression in older adults. Annu Rev Public Health. 2012;33:123-35.

11. Moher D, Liberati A, Tetzlaff J, Altman DG, Group P. Preferred reporting items for systematic reviews and meta-analyses: the PRISMA statement. J Clin Epidemiol. 2009;62(10):1006-12.

12. Moher D, Shamseer $L$, Clarke M, Ghersi D, Liberati A, Petticrew $M$, et al. Preferred reporting items for systematic review and meta-analysis protocols (PRISMA-P) 2015 statement. Syst Rev. 2015;4:1.

13. Ruddy R, House A. Psychosocial interventions for conversion disorder. Cochrane Database Syst Rev. 2005(4):CD005331. 
14. Cumpston M, Li T, Page MJ, Chandler J, Welch VA, Higgins JP, et al. Updated guidance for trusted systematic reviews: a new edition of the Cochrane Handbook for Systematic Reviews of Interventions. Cochrane Database Syst Rev. 2019;10:ED000142.

\section{Supplementary Files}

This is a list of supplementary files associated with this preprint. Click to download.

- AppendixASearchstrategy.pdf

- completedPRISMAPchecklist.docx 